\title{
Subject Review: Brain Tumor Detection Techniques
}

\author{
Wedad Abdul Khuder Naser \\ Department of Computer Science \\ Mustansiriyah University, College of Education \\ Baghdad-Iraq
}

\begin{abstract}
A brain tumor is one of the main causes of increased mortality among children and adults. The tumor is a major problem that is out of control over the normal force that regulates growth. There are several techniques for segmenting and detecting a brain tumor area on MRI images. In this paper, we provide background reviews of several proposed techniques for the recognition of brain tumors. There is a lot of literature on detecting this type of brain tumor and improving detection accuracy.
\end{abstract}

Key Words: Brain tumor, MRI images, Segmenting, Detection accuracy.

\section{INTRODUCTION}

Brain is an organ that control activities of all the part of the body. Abnormal brain cells growth leads to a brain tumor. Diagnosing a brain tumor is very important nowadays. A brain tumor is more curable and treatable if it is detected at an early stage. There are a number of imaging techniques used in the diagnosis and treatment of brain tumors, such as computed tomography (CT) and magnetic resonance imaging (MRI) [1]. MRI images are widely used for high-quality imaging mostly in brain scans because a brain tumor can be easily tracked through these images. MRI provides a digital representation of tissue characteristics that can be obtained at any tissue level. The images produced by the MRI scanner are sliced, and the added advantage is segmentation into both horizontal and vertical planes. These MRI scans are useful in easily identifying, detecting and classifying tumor parts in the brain easily [2].

Brain tumor is classified based on the type of tissue involved in the brain, the location of the tumor in the brain, which is a benign tumor or metastasis and various other considerations[3]. In the recent year, proposed several techniques for detecting a brain tumor. In this research, there are different techniques for brain tumors that help researchers choose the best techniques for detecting brain tumors.

\section{LITERATURE SURVEY}

Many studies on brain tumor detection techniques have been proposed. The following is a review of some of these studies:

In this paper [2], automatic brain tumor diagnosis using Convolutional Neural Networks (CNN) classification is proposed. There are six basic steps in the proposed approach. The sensitivity of the filter is reduced by smoothing the convolution filter (i.e. subsampling), the signal transfers from one layer to another layer is controlled by the activation layer, and the signal transfers from one layer to another layer is controlled by the activation layer. Using rectified linear units (RELU), the neurons in the preceding layer are connected to every neuron in the subsequent layer, and a loss layer is added at the conclusion of the training to provide feedback to the neural network. The accuracy is high and the complexity is low.

In this paper [3], a hybrid method employing a genetic algorithm (G A) and a support vector machine (SVM) for categorizing tumor tissue in magnetic resonance imaging (MRI) images is given. A wavelet-based texture's feature set is derived. The spatial gray level dependency method is used to extract optimal texture features from normal and malignant areas (SGLDM). The SVM classifier receives these features as input. GA is used to overcome a significant challenge in classification techniques: the selection of features. These ideal characteristics are used to categorize brain tissue as normal, benign, or cancerous. On a series of brain tumor photos, the algorithm's performance is assessed. 
In this paper[4], the fuzzy based control theory is applied for brain tumor segmentation and classification. The Fuzzy Interference System (FIS) is a specialized methodology for segmenting the brain. Supervised categorization is used to generate a membership function for a fuzzy controller. The performance is high, but the accuracy is low.

In this paper [5], fuzzy C-Means (FCM) segmentation is used to distinguish between tumor and non-tumor brain regions. Wavelet features are frequently extracted using a multilevel Discrete Wavelet Transform (DWT). Finally, a Deep Neural Network (DNN) is employed to accurately classify brain cancers. This method is compared to KNN, Linear Discriminant Analysis (LDA), and Sequential Minimal Optimization as classification approaches (SMO). The accuracy rate of DNN-based brain tumor categorization was 96.97 percent in the study. The complexity, on the other hand, is high, and the performance is low.

In this paper [6] , proposed that we focus on classifying mangoes based on their shape. They first extract the mango images' area of interest, then calculate the height and width of the various mango species extracted from the images. Principal component analysis, linear discriminant analysis, and nonlinear discriminant approaches are used to analyze training data using ROI.

In this paper [7], a seeded tumor segmentation approach is described, and it is compared to a graph cut-based segmentation method using the new Cellular Automata(CA) technique. Seed selection and Volume Of Interest (VOI) are computed for efficient brain tumor segmentation. This study also supports tumor cut segmentation. The level of complexity is low. However, the accuracy is low.

In this paper [8], DWT and probabilistic neural networks were used to identify and classify brain MRI images. MRI images include a variety of imaging techniques for examining the interior cells of the human brain. They first employ morphological processes to segment the brain tumor image, then utilize DWT to extract features from the segmented tumor. The features are then input into the PNN classifier, which is a technology for rapid and accurate brain tumor diagnosis when compared to the manual detection technique used by clinical experts.

In this paper [9], the purpose of the research study is to use image processing to segment tumorous tissue from brain MRI images. Image Analysis, Correction, Sensitivity, Sensitivity, and Statistics are the different categories. The BRATS database was used to review different algorithms for brain tumor segmentation using magnetic resonance imaging (MRI) of astrocytomas. For tumor tissue segmentation, the global baseline threshold method is used. Shape-based features are extracted in the feature extraction process. The KNN classifier divides images into two categories: low score and high score. Evaluation of the system based on parameters, severity, and characteristics.

In this paper[10], the local independent, projection-based classification (LIPC) method is used to classify the voxel of the brain. The path feature is also extracted using this method. As a result, LIPC eliminates the need for explicit regularization. The accuracy is low.

In this paper [11]. magnetic resonance imaging (MRI) was used to detect and retrieve a brain tumor. Images are gathered from the Internet, and samples from the radiologist are taken. The radiologists' images are converted into regular RGB images before being sent to a DICOM converter. The method is divided into three stages, with the first stage preprocessing the input image, the second stage threshold segmentation with further morphological processes, and the third stage tumor detection and extraction, with the image presented as an output. It uses a binarized image for automatic region splitting based on a region-growing technique, similar to how the automated classified sel works.

In this paper [12] , a new brain tumor segmentation system has been implemented, also known as a multimodal brain tumor segmentation scheme. In addition, different segmentation algorithms are being combined to get higher performance than the existing methods. However, the level of complexity is high.

in this paper[13], the suggested work is known as Image segmentation is the process of segmenting an image in order to extract different objects. The research presents a novel two-stage segmentation method for MRI brain tumors using a Gabor filter and contour level set segmentation. The contour level set approach is used to detect different regions in an image. This method allows us to extract only the tumor part, which is useful for further diagnostics analysis such as tumor size, shape, and density. The pixel values of tumor tissue are higher than those of normal tissue. The tumor's area and shape matrix were calculated. If the metric value is 1 , the tumor is benign; if the metric value is more, the tumor is malignant. 
In this paper[14], the proposed method consists of several steps, including dataset acquisition, preprocessing, segmentation using the Expectation Maximization (EM) algorithm and adaptive thresholding, feature extraction from an MRI data set using the Fast Fourier Transform (FFT), feature selection using the Minimal-Redundancy-Maximal-Relevance criterion (MRMR) to select the most valuable features, and classification.

In this paper[15], a novel bio-physiomechanical tumor growth model is presented to study the step-by-step tumor growth of patients. To avoid the significant tumor mass effect, it will be used with individual margins for gliomas and solid tumors. Combining discrete and continuous approaches, a tumor growth model is developed. The proposed scheme, which is based on atlas-based registration, offers for the tacit segmentation of tumor-bearing brain images. This method is commonly used to segment brain tissue. The computation time, on the other hand, is high.

In this paper[16], new multi-fractal (MultiFD) feature extraction and improved AdaBoost classification techniques are used to detect and segment the brain tumor. The texture of brain tumor tissue is retrieved using the MultiFD feature extraction technique. Improved AdaBoost classification methods are utilized to detect whether a given brain tissue is tumor or non-tumor tissue. The complexity is high.

In this paper[ 17], the results of a survey and segmentation of brain tumors are provided. Region-based segmentation, threshold-based segmentation, fuzzy C Means segmentation, Atlas-based segmentation, Margo Random Field (MRF) segmentation, deformable model, and geometric deformable model are all discussed. Precision, robustness, and validity are all assessed for each method.

In this paper[ 18], adaptive histogram equalization is used to improve the image's contrast. After that, Fuzzy CMeans (FCM) is used to segment the tumor from the rest of the brain image. After that, the Gabor feature is retrieved in order to filter out the aberrant brain cells. Finally, the fuzzy with K Nearest Neighbor (KNN) classification is employed to locate the anomaly in the brain MRI picture. The level of complexity is high. But, the precision is low.

In this paper[19], hybrid feature selection with ensemble classification is used to diagnose brain cancers. The GANNIGMAC, decision Tree, Bagging $\mathrm{C}$ based wrapper approach is used to obtain the decision rules. Also, employ hybrid feature selection to simplify the decision rules (GANNIGMAC + MRMR C+ Bagging C + Decision Tree).

In this paper[20], to detect brain cancers, they presented a hybrid method that employs statistical characteristics and a Fuzzy Support Vector Machine classifier. The proposed approach consists of four steps. In the first step, noise was reduced using an anisotropic filter. In the second stage, texture features collected from MR images are utilised. In the third phase, utilizing principal component analysis, the features of MR images were reduced to the most fundamental features. Finally, the tumor was classified as normal or abnormal using a Supervisor classifier-based Fuzzy Support Vector Machine. The accuracy of the classification was $95.80 \%$.

\section{CONCLUSIONS}

The main goal of this research article is to find efficient automatic brain tumor classification with high accuracy, performance and low complexity. In this research article, reviewed various techniques for Brain Tumor Detection . These techniques are studied wholly to find out which of these techniques is more efficient, accurate and less complicated . The research article showed that the Brain Tumor Classification Using Convolution Neyral Network (CNN) is more efficient with high accuracy and low complexity

\section{REFERENCES}

[1 ] Amit M., Yee K. T., Stephen J., Michael A., and Frederik L., "An Introduction to Tumor Brain Imagining “, Springer, 2014

[2] J.Seetha and Selvakumar Raja . , "Brain Tumor Classification Using Convolution Neural Network", Biomedical \& Pharmacology Journal, September 2018, Vol 11(3), p . 1457-1461

[3] Ahmed K., Karim G., "Hybrid Approach for Automatic Classification of Brain MRI Using Genetic Algorithm and Support Vector Machine “, Leonardo Journal of Sciences, pp.71-82,2010. 
[4] Janani and P. Meena, "image segmentation for tumor detection using fuzzy inference system", International Journal of Computer Science and Mobile Computing, 2(5): 244 - 248 (2013).

[5] Meiyan Huang et al, "Brain Tumor Segmentation Based on Local Independent Projection-based Classification", IEEE Transactions on Biomedical Engineering, IEEE, (2013).

[6] R. Karuppathal and V. Palanisamy, "Fuzzy based automatic detection and classification approach for MRI-brain tumor", ARPN Journal of Engineering and Applied Sciences, 9(12): (2014).

[7] Marco Alfonse and Abdel- Badeeh M. Salam, “ An Automatic Classification of Brain Tumor through MRI Using Support Vector Machine . “, Egyptian Computer Science Journal(ISSN:1110-2586), Volume 40-ISSUE 03, September 2016.

[8] Heba Mohsen et al, "Classification using Deep Learning Neural Networks for Brain Tumors", Future Computing and Informatics, pp 1-4 (2017)

[9] Abbas, Qaiser, et al “ Mango Classification Using Texture \& Shape Features “, International Journal of computer science and network security 18.8:132-138,2018.

[10] Anjali, V. Charan, and Shanthi Prince. , “A novel methodology for brain tumor detection based on two-stage segmentation of MRI images .", Advanced Computing and Communication System, International Conference on IEEE,2015.

[11] AndacHamamci et al, "Tumor-Cut: Segmentation of Brain Tumors on Contrast-Enhanced MR Images for Radiosurgery Applications”, IEEE Transactions on Medical Imaging, 31(3): (2012).

[12] Stefan Bauer et al, "Multiscale Modeling for Image Analysis of Brain Tumor Studies", IEEE Transactions on Biomedical Engineering, 59(1): (2012)

[13] Shree, N. Varuna, and T.N.R. Kumar. "Identification and classification of brain tumor MRI image with feature extraction using DWT and probabilistic neural network.” Brain informatics 5.1:23-30, 2018.

[14] Gupta, Shefali, et al " Segmentation, Feature Extraction and Classification of Astrocytoma in MR Images ." Indian Journal of Science and Technology 9.36:1-8, 2016.

[15] Isselmou, A., Shuai Zhang, and Guizhi Xu "A novel approach for brain tumor detection using MRI images ." J Biomed Sci Eng. 9:44-52,2016.

[16] Bjoern H. Menze et al, "The Multimodal Brain Tumor Image Segmentation Benchmark (BRATS)”, IEEE Transactions on Medical Imaging, (2014).

[17] Atiq Islam et al, "Multi-fractal Texture Estimation for Detection and Segmentation of Brain Tumors", IEEE, (2013).

[18] Shamsul Huda et al, "A Hybrid Feature Selection with Ensemble Classification for Imbalanced Healthcare Data: A Case Study for Brain Tumor Diagnosis”, IEEE Access, 4: (2017).

[19] Jin Liu et al, "A Survey of MRI-Based Brain Tumor Segmentation Methods", TSINGHUA Science and Technology, 19(6) (2011).

[20] A. Jayachandran and R. Dhanasekaran, "Brain Tumor Detection And Classification of MR Images Using Texture Features And Fuzzy SVM Classifier ", Research Journal of Applied Sciences, Engineering and Technology 6(12), 2264-2269, 2013. 